\title{
Ultrasound-Guided Near-Infrared Optical Tomography
}

National Cancer Institute

\section{Source}

National Cancer Institute. Ultrasound-Guided Near-Infrared Optical Tomography. NCI

Thesaurus. Code C128880.

An imaging modality that combines ultrasound imaging with diffused light imaging by near-infrared (NIR) optical tomography to obtain coreg istered ultrasound and NIR diffusive light images in order to improve optical imaging reconstruction and location certainty. The use of NIR in this combination can also be used to assess functional parameters of a tissue. 